\title{
Hallazgo de restos de la serpiente Dinilysia patagonica Smith-Woodward 1901 en una nueva localidad del Santoniano de Patagonia, Argentina
}

\author{
Record of remains of the snake Dinilysia patagonica \\ Smith-Woodward 1901 in a new locality of the Santonian \\ of Patagonia, Argentina
}

\author{
L.N. Triviño ${ }^{1}$, A.M. Albino ${ }^{2}$ \\ 1 CONICET - Sección Herpetología, Museo de La Plata, Universidad Nacional de La Plata, Paseo del Bosque \\ s/nº, B1900FWA La Plata, Argentina. Email: lauratrivinio@fcnym.unlp.edu.ar \\ 2 CONICET- Departamento de Biología, Universidad Nacional de Mar del Plata, Funes 3250, B7602AYJ Mar del Plata, \\ Argentina
}

\section{RESUMEN}

En este trabajo se describen nuevos restos de la serpiente cretácica Dinilysia patagonica recuperados en la localidad Barreales Norte, Formación Bajo de la Carpa (Santoniano superior), provincia del Neuquén, Argentina. El material comprende la parte posterior de un cráneo parcialmente conservado y articulado con un fragmento del hueso compuesto, atlas y axis. Además, se preservaron seis secciones de columna vertebral con vértebras precloacales. La asignación a Dinilysia patagonica se basa en: cráneo de tamaño grande, otooccipital con largo proceso paraoccipital adosado a un elongado supratemporal que se extiende más allá del nivel del cóndilo occipital; cresta sagital del parietal bien desarrollada y continua con la cresta del supraoccipital; proótico en forma de I cursiva; vértebras anchas, cortas y robustas; prezigapófisis largas y muy inclinadas sobre la horizontal; canal neural pequeño y trilobulado; proceso prezigapofisario reducido; arcos neurales aplanados con el margen posterior recto. Este material agrega una nueva localidad al norte del río Neuquén para la distribución de la especie en el Cretácico superior.

Palabras clave: Dinilysia; Cretácico; Argentina; Serpientes.

\section{ABSTRACT}

In this study, we describe new remains of the cretaceous snake Dinilysia patagonica from beds of the locality Barreales Norte, Bajo de la Carpa Formation (Upper Santonian), Neuquén province, Argentina. The material comprises the back of a partially preserved skull, articulated with a fragment of the compound bone, atlas and axis. Additionally, six fragments of precloacal vertebrae have been preserved. The assignation to Dinilysia patagonica is based on: large skull, otooccipital with long paraoccipital process adjacent to an elongate supratemporal that

Recibido el 7 de agosto de 2014 / Aceptado el 15 de abril de 2015 / Publicado online el 2 de octubre de 2015

Citation / Cómo citar este artículo: L.N. Triviño \& A.M. Albino (2015). Hallazgo de restos de la serpiente Dinilysia patagonica SmithWoodward 1901 en una nueva localidad del Santoniano de Patagonia, Argentina. Estudios Geológicos 71(2): e033. http://dx.doi. org/10.3989/egeol.41858.347.

Copyright: () 2015 CSIC. This is an open-access article distributed under the terms of the Creative Commons Attribution-Non Commercial (by-nc) Spain 3.0 License. 
extends farther the level of the occipital condyle; well developed sagital crest of parietal bone, continuously joined with the supraoccipital crest; italics I-like prootic; wide, short and robust vertebrae; prezygapophyses long and highly inclined above the horizontal; triangular section of vertebral centrum; reduced prezygapophyseal process; flattened neural arches with straight posterior edge. This material adds a new Upper Cretaceous location for the distribution of the species at the north of the Neuquén River.

Keywords: Dinilysia; Cretaceous; Argentina; Snakes.

\section{Introducción}

Las serpientes son conocidas en territorio sudamericano desde el Mesozoico. Restos de este clado son comunes y diversos en depósitos cretácicos de Patagonia, incluyendo algunas de las más primitivas serpientes terrestres como Najash rionegrina del Cenomaniano-Turoniano (Apesteguía \& Zaher, 2006; Zaher et al., 2009; Palci et al., 2013) y Dinilysia patagonica del Santoniano-Campaniano (SmithWoodward, 1901; Estes et al., 1970; Hecht, 1982; Rage \& Albino, 1989; Caldwell \& Albino, 2002; Scanferla \& Canale, 2007; Caldwell \& Calvo, 2008; Zaher \& Scanferla, 2012, Scanferla \& Bhullar, 2014; Palci \& Caldwell, 2014). Además, varias especies de Madtsoiidae, el "anilioideo" Australophis anilioides y formas inciertas de serpientes se reconocen para el Campaniano-Maastrichtiano (Albino, 1986, 1994, 2000, 2007, 2011; Martinelli \& Forasiepi, 2004; Gómez et al., 2008; Gómez, 2011). Entre todas estas serpientes se destaca $D$. patagonica, representada por una cantidad relevante tanto de restos craneanos como vertebrales procedentes de localidades de las provincias de Río Negro (Cinco Saltos y Paso Córdova) y Neuquén (Aguada Toledo, Barreales Norte, ciudad de Neuquén y Puesto La Rinconada) (Rage \& Albino, 1989; Bonaparte, 1991; Caldwell \& Albino, 2002; Albino \& Caldwell, 2003; Albino, 2007; Scanferla \& Canale, 2007; Caldwell \& Calvo, 2008; Zaher \& Scanferla, 2012; Filippi \& Garrido, 2012). Entre estos materiales, el procedente de Barreales Norte (Figura 1), consistente en un cráneo incompleto y secciones de columna vertebral, aún se encuentra inédito. En el presente trabajo se realiza la descripción y discusión de este material que proviene de una localidad al Norte del río Neuquén.

\section{Materiales y métodos}

Además del uso de la bibliografía específica, el material estudiado (MCF-PVPH 517) fue comparado directamente con ejemplares de Dinilysia patagonica: MLP 26-410 (holotipo), MLP 79-II27-1 al 79-II-27-17, MACN-N 25, MACN-N 26, MACN-N 27, MACN-N 104, MACN-N 115, MACN-N 116, MACN-RN 976, MACN-RN 1013, MACN-RN 1014, MACN-RN 1015, MACN-RN 1016, MACN-RN 1017, MACN-RN 1018, MACN-RN 1019, y MACN-RN 1021. Igualmente, se utilizaron materiales craneanos y postcraneanos comparativos de los escamosos actuales Boa constrictor occidentalis, Eunectes notaeus, Salvator merianae, Teius oculatus, Broghammerus reticulatus, Philodryas olfersii y Bothrops alternatus.

\section{Abreviaturas institucionales:}

MACN: Museo Argentino de Ciencias Naturales "Bernardino Rivadavia", Buenos Aires

MCF-PVPH: Museo Carmen Funes, Plaza Huincul. MLP: Museo de La Plata, La Plata.

Abreviaturas anatómicas: $\mathrm{H}$, altura de la vértebra desde la base del cóndilo hasta el extremo dorsal de la espina neural; po-po, distancia entre los extremos de las postzigapófisis; pr-po, distancia interzigapofisaria (entre el extremo de la prezigapófisis y el de la postzigapófisis).

\section{Paleontología Sistemática}

Orden SQUAMATA Oppel, 1811

Suborden SERPENTES Linnaeus, 1758

Género Dinilysia Smith-Woodward, 1901

Especie tipo: Dinilysia patagonica SmithWoodward, 1901; designación original. Santoniano de la provincia del Neuquén.

Material: MCF-PVPH 517. Porción posterior de un cráneo articulado, incluyendo un fragmento del hueso compuesto izquierdo. Articula con fragmentos del atlas y axis. En el mismo bloque sedimentario se conservaron seis secciones con vértebras precloacales articuladas. 


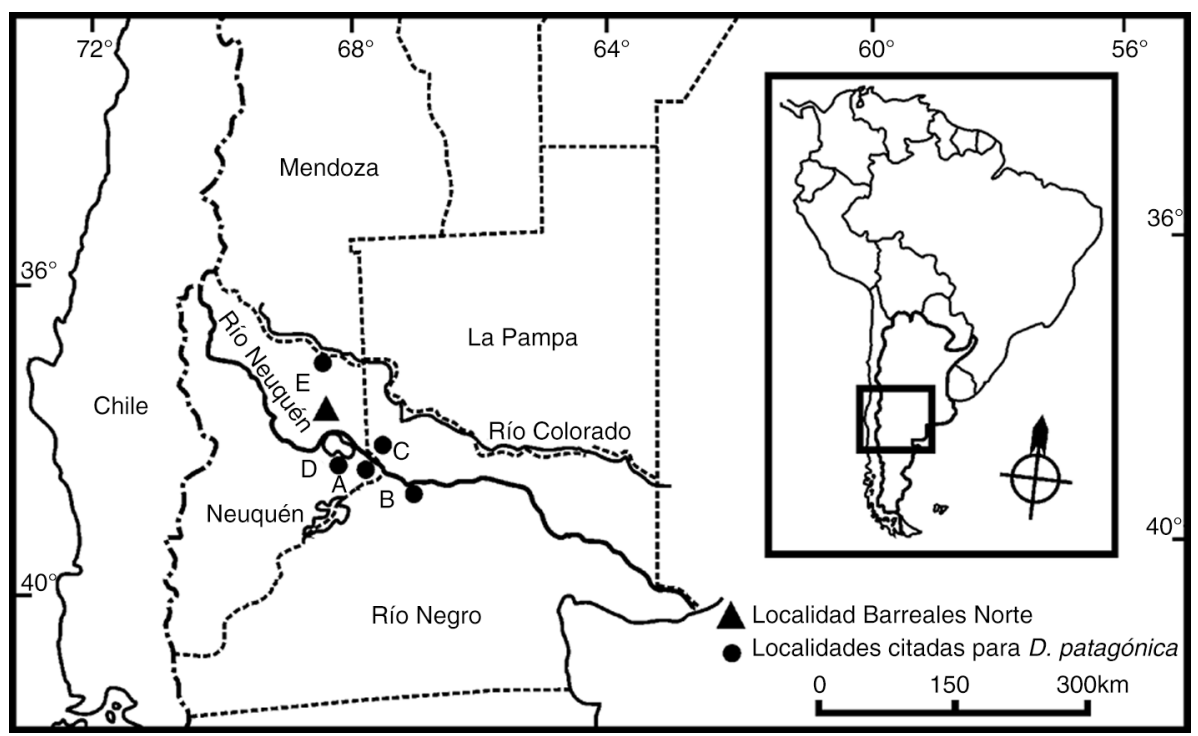

Fig. 1.-Mapa de distribución de Dinilysia patagonica. El triángulo indica la localidad Barreales Norte. Los círculos indican localidades donde se han citado ejemplares de Dinilysia A, Ciudad de Neuquén; B, Paso Córdova; C, Cinco Saltos; D, Aguada Toledo; E, Rincón de los Sauces.

Procedencia geográfica: Barreales Norte, aproximadamente a $45 \mathrm{Km}$. al sur del volcán Auca Mahuida, provincia del Neuquén, Argentina.

Procedencia estratigráfica: Grupo Neuquén, Subgrupo Río Colorado, Formación Bajo de la Carpa (Santoniano, Cretácico Superior).

Descripción: La conservación de los restos tanto craneanos como vertebrales es deficiente. Del cráneo sólo se conserva la mitad posterior, principalmente el parietal que es angosto en su extremo más anterior y se expande transversalmente a la altura de la región ótica (Figuras 2A y 3). Sobre el lado izquierdo contacta con el proótico y supratemporal; dorsodistalmente con el supraoccipital mediante una sutura transversal irregular y a los lados de la misma con los otooccipitales por medio de una proyección en forma de cuña. En la línea medio dorsal, se observa la cresta sagital que está rota y erosionada pero puede notarse que era alta. Se conserva parte del proótico izquierdo, relativamente grande, representado por su mitad dorsal y el proceso anterodorsal que limita el margen dorsal del foramen trigémino. La pared descendente del parietal no llega a visualizarse si participa en el margen anterior de dicho foramen. Posteriormente el proótico está muy deteriorado. La región ótica, observada en vista ventral, se conserva como una cavidad elipsoidal sobre el lateral derecho, elongada anteroposteriormente, que concuerda con la posición de la cavidad del oído medio. Se preservó parcialmente el supratemporal izquierdo, de forma lanceolada, extendido más allá del nivel del cóndilo occipital acompañando el desarrollo posterior del proceso paraoccipital. En vista lateral contacta anteroventralmente con el proótico y posteroventralmente con el cuadrado. El supraoccipital está completamente conservado, es un hueso posterodorsal impar, corto y ancho. Limita anteriormente con el parietal y caudalmente con los otooccipitales. En la zona media presenta una cresta anteroposterior muy erosionada continua con la cresta sagital del parietal. A ambos lados se aprecian crestas muy marcadas que nacen en el borde más anterior del hueso y se extienden posterolateralmente, siendo especialmente protuberantes en los extremos laterales. Los otooccipitales se ubican en el extremo posterodorsal del cráneo; se observa especialmente el izquierdo que limita anteriormente con el supraoccipital y parietal, y lateralmente con el supratemporal. El expandido proceso paraoccipital izquierdo está bien conservado, supera distalmente el nivel del cóndilo occipital y se inclina dorsomedialmente. Medialmente, una corta expansión del supraoccipital se introduce entre los otooccipitales. 


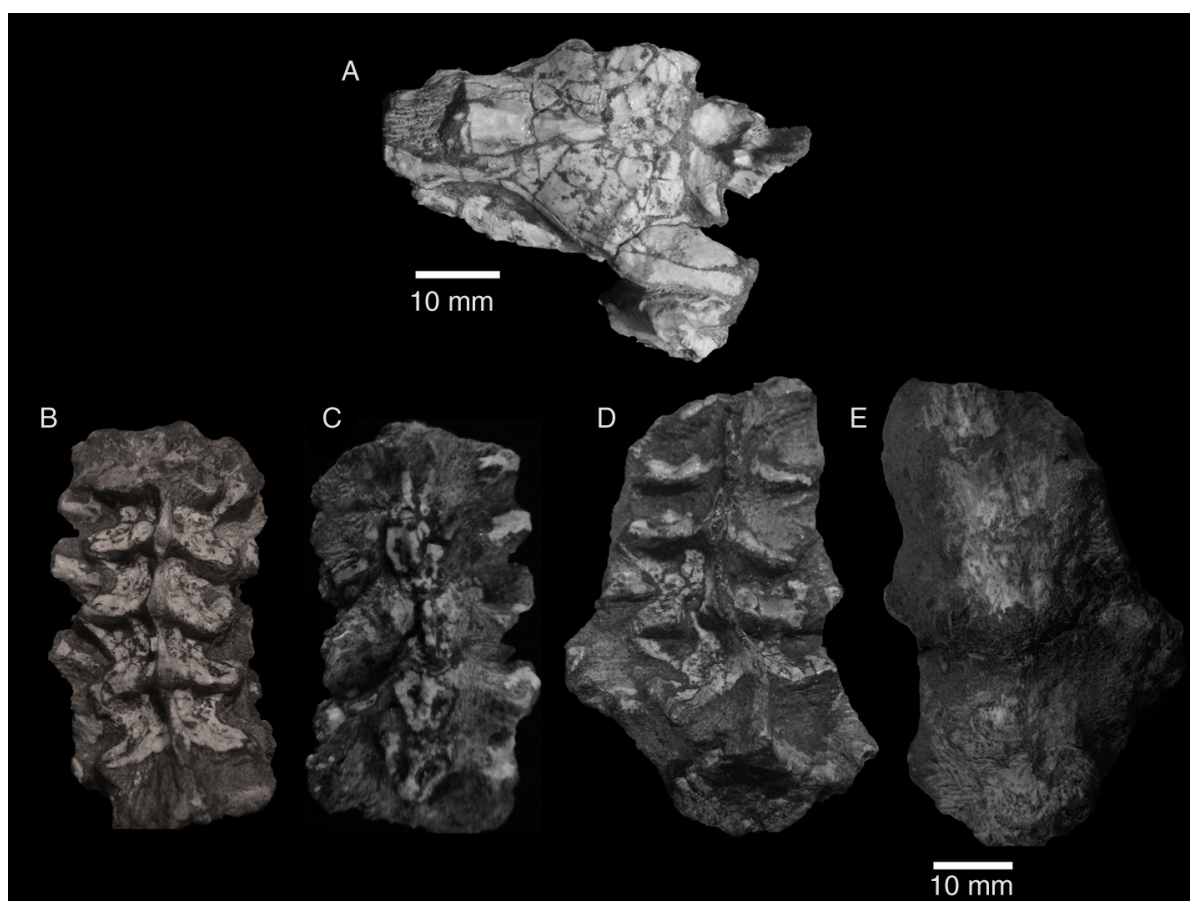

Fig. 2.-Cráneo en vista dorsal (A); secciones vertebrales MCF-PVPH 517-4 en vista dorsal (B) y ventral (C); MCF-PVPH 517-5 en vista dorsal (D), ventral (E).

En vista ventral, se ha conservado el lateral izquierdo del basioccipital, pero muy deteriorado; posteromedialmente se angosta formando la base del cóndilo occipital. El basiparasfenoides se conservó cruzado por dos fracturas que desplazaron el fragmento medio hacia la derecha, anteriormente a los procesos basipterigoideos. Dicho segmento desplazado conservó un fragmento del canal vidiano. Formando parte del arco palato-maxilar, el palatino izquierdo se ha conservado en varios fragmentos no continuos, uno de los cuales articula con el pterigoides. El único pterigoides conservado es el izquierdo. En la superficie ventral presenta el proceso interno que articula con el proceso basipterigoideo izquierdo.

El cuadrado izquierdo es el único conservado y está casi completo; es grande, trirradiado, robusto, y corto, articulado dorsalmente con el supratemporal. Se observan claramente dos de sus tres ejes, de los cuales el eje posteroventral contacta con la superficie articular del hueso compuesto, mientras que el posterodorsal, que es el más robusto, corre paralelo al supratemporal extendiéndose caudalmente más allá del nivel del cóndilo occipital.

De la mandíbula se conservó un fragmento posterior del hueso compuesto izquierdo articulado al cuadrado. En el extremo anterior del hueso compuesto se observa una pequeña cresta, poco marcada, que representa a la cresta surangular de la fosa aductora.

Con respecto al esqueleto postcraneano, se preservaron también fragmentos de atlas y axis articulados al cráneo. El atlas está representado por dos medios arcos neurales grandes y bien conservados, que habrían estado unidos dorsalmente en la línea media, mientras que el axis está muy deteriorado, sin características que resaltar. Sin articular con el material craneal también se conservaron seis secciones de vértebras articuladas (MCF-PVPH 517-1 a MCF-PVPH 517-6) (Figura 2B-E y 4) entre las cuales se identificaron vértebras precloacales medias y posteriores (sensu Rage \& Albino, 1989). Cada sección contiene una cantidad variable de vértebras: MCF-PVPH 517-1 y MCF-PVPH 517-4 incluye seis vértebras dorsales medias cada una, mientras que MCF-PVPH 517-5 contiene cinco vértebras dorsales posteriores. Los bloques MCF-PVPH 517-2, MCF-PVPH 517-3 y MCF-PVPH 517-6 presentan tres vértebras cada una sin poder establecerse la ubicación en la columna vertebral. Algunas de las vértebras están articuladas con la parte proximal de sus respectivas costillas. 

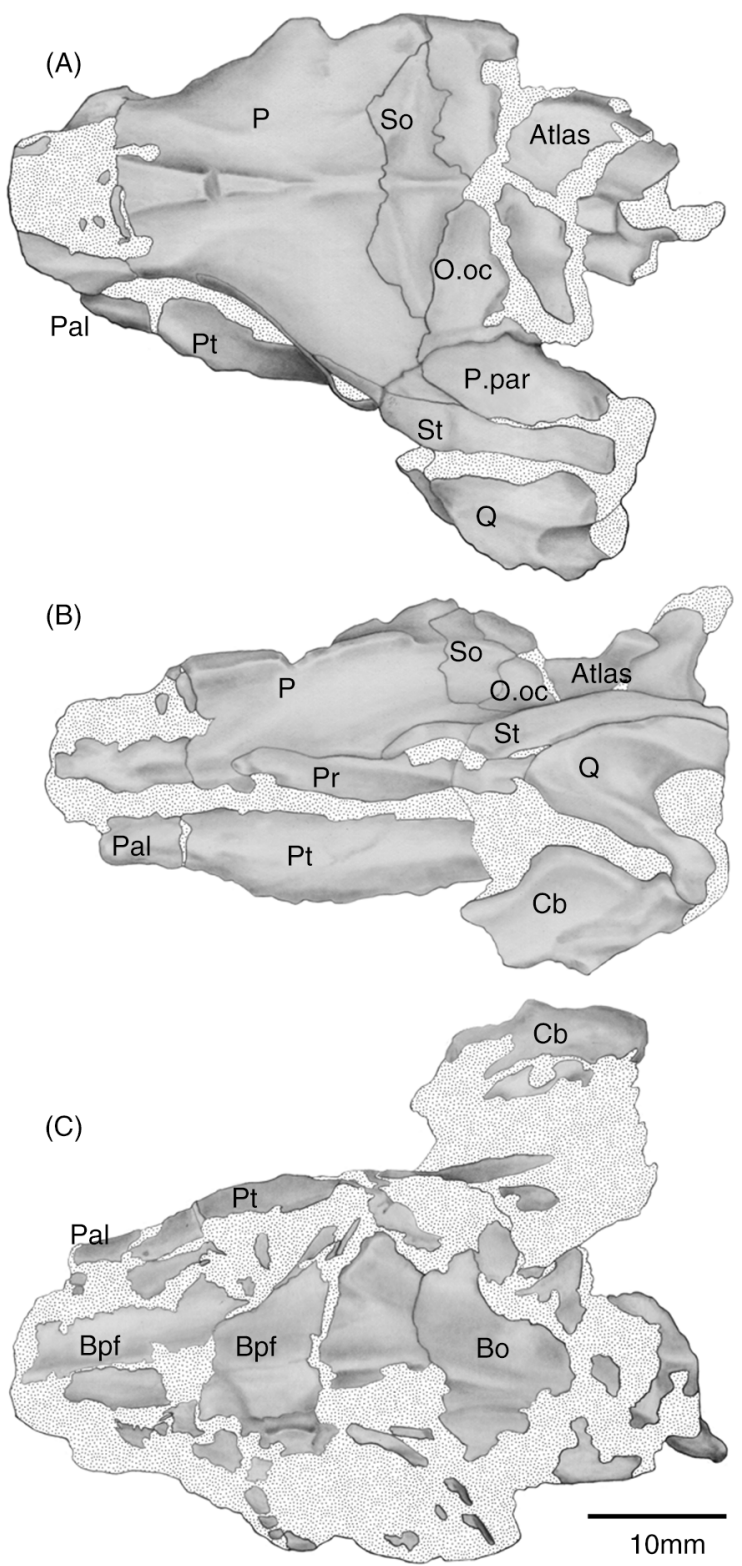

Fig. 3.-Porción posterior del cráneo articulado (MCF-PVPH 517) en vistas dorsal (A), lateral izquierda (B) y ventral (C). Abreviaturas: Bsf, basiparasfenoides; Bo, basioccipital; $\mathrm{Cb}$, hueso compuesto; Oo.c, otooccipital; Pal, palatino; P, parietal; P. par, proceso paraoccipital; Po, proceso paraoccipital; Pr, proótico; Pt, pterigoides; Q, cuadrado; So, supraoccipital; St, supratemporal.

En general las vértebras son de tamaño mediano, con una distancia entre postzigapófisis cercana a la de un ejemplar de Boa constrictor occidentalis cuya longitud hocico-cloaca era de $187 \mathrm{~cm}$ y la longitud total de $205 \mathrm{~cm}$. Son vértebras más anchas que altas (po-po $>$ H) y que largas (po-po>pr-po), y presentan un grado de conservación variable pero las

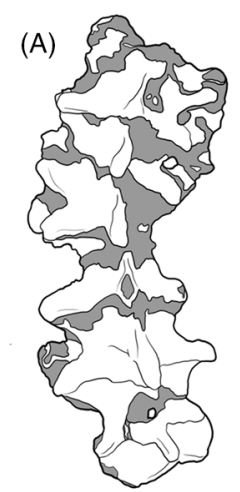

(B)

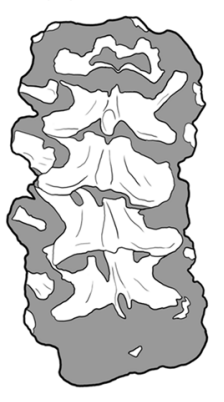

$30 \mathrm{~mm}$

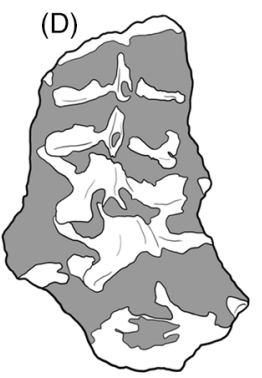

(E)

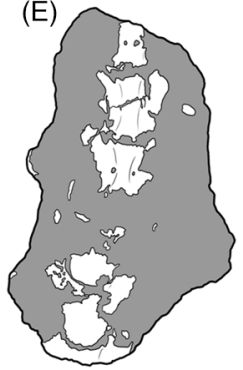

(C)

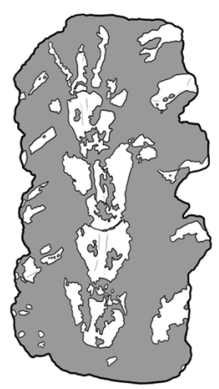

(F)

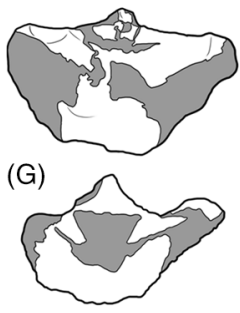

Fig. 4.-Secciones con vértebras precloacales articuladas. Sección MCF-PVPH 517-1 en vista dorsal (A), MCF-PVPH 517-4 en vista dorsal (B) y ventral (C), MCF-PVPH 517-5 en vista dorsal $(D)$, ventral $(E)$, anterior $(F)$ y posterior $(G)$.

características generales en todas las secciones son homogéneas desde el punto de vista taxonómico, de manera que puede establecerse que pertenecen a una misma especie. Además, la asociación original en un mismo bloque sedimentario donde se encontraba también el cráneo articulado indica que se trataría de un único individuo. En vista dorsal se observa que el arco neural es ancho y presenta el borde posterior rectilíneo; la espina neural nace sobre el techo del zigósfeno y se engrosa posteriormente sobrepasando el borde posterior del arco neural; al menos en uno de los fragmentos puede notarse que el zigósfeno tiene borde anterior cóncavo; tanto prezigapófisis como postzigapófisis presentan la superficie de articulación larga y ovalada y desarrollo más bien lateral; la constricción interzigapofisaria es corta y profunda; el proceso prezigapofisario es corto, agudo, y sobrepasa ligeramente el extremo prezigapofisario. La vista anterior sólo puede observarse parcialmente en los bloques MCF-PVPH 517-1 y MCF-PVPH 517-5. El arco neural es bajo y deprimido; el canal neural es pequeño y de contorno trilobulado; y las 
prezigapófisis son largas y están fuertemente inclinadas sobre la horizontal alcanzando el nivel del techo del zigósfeno. La presencia de forámenes paracotilares es dudosa debido a la mala conservación. La vista posterior también se observa parcialmente. Se destaca el deprimido arco neural y el cóndilo semiesférico; el zigantro se encuentra relleno de sedimento; y las postzigapófisis son largas e inclinadas sobre el plano horizontal. En la vista lateral se distingue la espina neural completamente conservada en algunas vértebras, es alta, larga e inclinada anteroposteriormente. El centro vertebral es corto y las paradiapófisis están poco diferenciadas en superficie diapofisaria y parapofisaria. En vista ventral el centro vertebral es de sección triangular, más ancho anterior que posteriormente; los forámenes subcentrales se ubican próximos al plano sagital; y la carena hemal está bien definida pero presenta desarrollo desigual en las secciones medias y posteriores.

Las vértebras medias se diferencian de las posteriores por presentar la constricción interzigapofisaria más profunda, la separación entre los extremos de pre y postzigapófisis menor y la espina neural más alta. En vista ventral, las vértebras medias presentan una carena hemal bien desarrollada, sobresaliente y delgada, mientras que en las posteriores la carena hemal es aplanada y ancha. Además, el centro vertebral es más robusto y el tamaño relativo del cóndilo es mayor en las vértebras posteriores respecto a las medias.

Se conservaron algunos fragmentos de costillas asociados a las secciones vertebrales. En la sección MCF-PVPH 517-1 se preservó un segmento proximal de costilla, en el cual se observa la lámina anteroventral y el proceso pseudotuberculiforme dorsal que completa el contacto con la paradiapófisis.

\section{Discusión}

El material estudiado se asigna a la especie extinta Dinilysia patagonica debido a la siguiente combinación de caracteres: otooccipital con largos procesos paraoccipitales extendidos más allá del nivel del cóndilo y adosados al supratemporal; cresta sagital del parietal bien desarrollada y continua con la cresta del supraoccipital; proótico en forma de I cursiva. Vértebras más cortas que anchas; zigapófisis largas y fuertemente inclinadas sobre el plano horizontal; proceso prezigapofisario corto; arco neural deprimido y con borde posterior rectilíneo; zigósfeno escotado; espina neural bien desarrollada; forámenes laterales presentes; forámenes subcentrales próximos al plano sagital; y costillas con proceso pseudotuberculiforme.

El material analizado en este trabajo permite realizar algunas observaciones complementarias a lo conocido sobre el cráneo de esta especie. Estes et al. (1970) designan como astilla del proótico lo que correspondería a un fragmento posterior del parietal que está separado del cuerpo principal del mismo por una fractura. Como se ha mencionado, en el cráneo descripto no se puede observar si el parietal interviene en la formación del margen anterior del foramen trigémino; sin embargo, durante la comparación de los diversos materiales de Dinilysia patagonica se advirtió que dicha intervención ocurre en los cráneos MLP 26-410, MLP 79-II-27-1, MACN-N 27 y MACN-RN 1014. El caso extremo de la participación del parietal en el foramen del trigémino se presenta en el cráneo de menor tamaño (MACN-N 104). En el caso de grandes ejemplares como MACN-RN 1015, el parietal queda excluido. Esta condición permite inferir que tal variación estaría vinculada a la edad del individuo. Esto es consistente con lo referido por Scanferla \& Bhullar (2014) respecto que se trataría una variación ontogenética postnatal. De esta manera, los cráneos comparativamente más pequeños, donde el parietal aún tiene participación en el margen del foramen trigémino, corresponderían a individuos jóvenes que aún no han alcanzado su tamaño máximo.

El supraoccipital del ejemplar estudiado, al igual que en MLP 79-II-27-1, presenta la sutura con el parietal en forma irregular, mientras que en el resto de los cráneos de Dinilysia la sutura es en línea recta. Esta diferencia podría deberse a problemas en la preservación o que exista variación intraespecífica.

Hasta comienzos de 1980 Dinilysia patagonica era conocida únicamente por el holotipo, el cual consiste en cráneo y vértebras asociadas (SmithWoodward, 1901; Estes et al., 1970; Hecht, 1982) proveniente de sedimentitas de la Formación Bajo de la Carpa aflorantes en la localidad Boca del Sapo, en las afueras de la ciudad de Neuquén, provincia del Neuquén (Figura 1A). Posteriormente Rage \& Albino (1989) describieron abundantes secciones 
de columna vertebral procedentes de la misma localidad y formación que el material tipo. A partir del 2000, los materiales que se citan en los diferentes trabajos provienen de nuevas localidades. Caldwell \& Albino (2002) y Caldwell \& Calvo (2008) analizan y discuten caracteres de Dinilysia incluyendo materiales recuperados en Paso Córdova, al sur del Río Negro en la provincia homónima (Figura 1B). Albino (2007) resume los hallazgos conocidos de Dinilysia y amplía el biocrón de la misma al Campaniano temprano sobre la base de nuevos materiales procedentes de la Formación Anacleto aflorantes en Cinco Saltos, provincia de Río Negro (Figura 1C). Posteriormente, Scanferla \& Canale (2007) describen vértebras medias-posteriores articuladas halladas también en la Formación Anacleto pero en la localidad Aguada Toledo, de la provincia del Neuquén (Figura 1D). Finalmente, Filippi \& Garrido (2012) dan a conocer nuevo material vertebral recuperado en niveles de la Formación Anacleto expuestos en la localidad Rincón de los Sauces, provincia del Neuquén (Figura 1E). De esta manera, los registros de Dinilysia se extienden desde el Santoniano al Campaniano temprano y se limitan a las provincias de Río Negro y Neuquén (Figura 1).

De los depósitos en que se han encontrado restos de Dinilysia también se han rescatado otros diversos tetrápodos. Según Leanza et al., 2004, las formaciones Bajo de la Carpa y Anacleto, que integran el subgrupo Río Colorado, poseen asociaciones de tetrápodos similares caracterizadas por terópodos de pequeño porte como alvarezsáuridos y abelisáuridos y grandes carnotaurinos como Abelisaurus comahuensis y Aucasaurus garridoi. Se han registrado titanosaurios desde pequeños, robustos y con armaduras como los saltasaurinos, hasta grandes y gráciles como los eutitanosaurios. Entre los ornitópodos está presente el euiguanodóntido basal de pequeño tamaño Gasparinisaura cincosaltensis. Las aves están representadas por enanthiornithinaes, a través de ignitas, huesos y nidos con huevos. Los animales más numerosos fueron los crocodyliformes notosuquidos siendo formas carnívoras terrestres y otros que merodeaban el agua. Se encontró un quélido relacionado con Chelus y pleurodiras vinculadas con podocnemididea. En esta asociación el escamoso dominante es la serpiente primitiva Dinilysia patagonica; sin embargo, un fragmento mandibular izquierdo con dientes de un lagarto Scincomorpha fue reconocido para la Formación Anacleto en la localidad de Cinco Saltos (Brizuela \& Albino, 2011). Es notable para todo el Mesozoico sudamericano la escasez de registros de lagartos en relación a la abundancia de serpientes reconocidas (Albino \& Brizuela, 2014), lo cual podría deberse a una situación vinculada con el origen y evolución de los primeros primordialmente en el hemisferio norte, o bien a un sesgo en el registro debido al tamaño, condiciones tafonómicas, orientación de las prospecciones paleontológicas, $\mathrm{u}$ otras causas.

El material descripto en este trabajo procedente de la localidad Barreales Norte, corrobora una nueva localidad santoniana para la distribución geográfica de la especie, ubicada al sur del Río Colorado y norte del Río Neuquén. Por el momento, sin embargo, los registros de esta serpiente se limitan al SantonianoCampaniano temprano del norte de la Patagonia argentina no existiendo referencias para ningún otro lugar en el mundo, lo cual, sumado a su condición primitiva y a su excelente preservación, la convierten en una especie particularmente significativa en el reconocimiento de la evolución del grupo.

\section{AGRADECIMIENTOS}

Se agradece a las autoridades del museo Carmen Funes y la Secretaría de Cultura de la provincia del Neuquén que facilitaron el material para su estudio. A M. Reguero (MLP) y S. Alvarez (MACN) por permitir el acceso a las colecciones paleontológicas. J. Williams facilitó los especímenes de comparación y brindó su apoyo incondicional. M. Sosa realizó las ilustraciones. Al CONICET por concederme una beca para la realización de este trabajo. Este trabajo forma parte del Proyecto Sistemática, Biogeografía y Ecología de Anfibios y Reptiles del Cono Sur de Sudamérica cod. 11/N690, acreditado por UNLP. PIP-CONICET 112-200901-00176.

\section{Referencias}

Albino, A.M. (1986). Nuevos Boidae Madtsoiinae en el Cretácico tardío de Patagonia (Formación Los Alamitos, Río Negro, Argentina). $4^{\circ}$ Congreso Argentino de Paleontología y Bioestratigrafía (Mendoza), Actas, 2:15-21.

Albino, A.M. (1994). Una nueva serpiente (Reptilia) en el Cretácico Superior de Patagonia, Argentina. Pesquisas, 21: 58-63.

Albino, A.M. (2000). New record of snakes from the Cretaceous of Patagonia (Argentina). Geodiversitas 22: $247-253$. 
Albino, A.M. (2007). Lepidosauromorpha. In: Patagonian Mesozoic Reptiles (Gasparini, Z., Salgado, L., Coria, RA., Eds.), Indiana, Indiana University Press, 87-115.

Albino, A.M. (2011). Evolution of Squamata Reptiles in Patagonia based on the fossil record. Biological Journal of the Linnean Society, 103: 441-457. http:// dx.doi.org/10.1111/j.1095-8312.2011.01691.x.

Albino, A.M. \& Caldwell, M.W. (2003). Hábitos de vida de la serpiente Cretácica Dinilysia patagonica Woodward. Ameghiniana, 40: 407-414.

Albino, A.M. \& Brizuela, S. (2014). An overview of the South American fossil Squamates. The Anatomical Record, 297 (3): 349-368. http://dx.doi.org/10.1002/ ar.22858.

Apesteguía, S. \& Zaher, H. (2006). A Cretaceous terrestrial snake with robust hindlimbs and a sacrum. Nature, 440: 1037-1040. http://dx.doi.org/10.1038/ nature 04413 .

Bonaparte, J.F. (1991). Los vertebrados fósiles de la Formación Río Colorado, de la ciudad de Neuquén y cercanías, Cretácico Superior, Argentina. Revista del Museo Argentino de Ciencias Naturales Bernardino Rivadavia, 4: 16-124.

Brizuela, S. \& Albino, A. (2011). A Scincomorpha lizard from the Campanian of Patagonia. Cretaceous Research, 32 (6): 781-785. http://dx.doi. org/10.1016/j.cretres.2011.05.006.

Caldwell, M.W. (1999). Squamate phylogeny and the relationships of snakes and mosasauroids. Zoological Journal of the Linnean Society, 125: 115-147. http:// dx.doi.org/10.1111/j.1096-3642.1999.tb00587.x.

Caldwell, M.W. \& Albino, A.M. (2003). Exceptionally preserved skeletons of the Cretaceous snake Dinilysia patagonica Woodward, 1901. Journal of Vertebrate Paleontology, 22: 861-866. http://dx.doi.org/10.167 1/0272-4634(2002)022[0861:EPSOTC]2.0.CO;2.

Caldwell, M.W. \& Calvo, J. (2008). Details of a new skull and articulated cervical column of Dinilysia patagonica Woodward, 1901. Journal of Vertebrate Paleontology, 28: 349-362. http://dx.doi. org/10.1671/0272-4634(2008)28[349:DOANSA] 2.0.CO;2.

Conrad, J.L. (2008). Phylogeny and systematics of Squamata (Reptilia) based on morphology. Bulletin of the American Museum of Natural History, 310: 1-182. http://hdl.handle.net/2246/5915.

Estes, R.; Frazzetta, T.H. \& Williams, E.E. (1970). Studies on the fossil snake Dinilysia patagonica Woodward: Part I. Cranial morphology. Bulletin of the Museum of Comparative Zoology 140: 25-73.

Filippi, L.S. \& Garrido, A.C. (2012). Nuevo registro del género Dinilysia (Squamata, Serpentes) para la formación Anacleto (Campaniano inferior-medio), Rincón de Los Sauces, Neuquén, Argentina. Ameghiniana, 49: 132-136.
Gómez, R.O. (2011). A Snake Dentary from the Upper Cretaceous of Patagonia. Journal of Herpetology, 45: 230-233. http://dx.doi.org/10.1670/10-048.1.

Gómez, R.O.; Báez, A.M. \& Rougier, G.W. (2008). An anilioid snake from the Upper Cretaceous of northern Patagonia. Cretaceous Research, 29: 481-488. http:// dx.doi.org/10.1016/j.cretres.2008.01.002.

Hecht, M.K. (1982). The vertebral morphology of the cretaceous snake Dinilysia patagonica Woodward. Neues Jarhbuch der Geologie und Paläontologie Monatshefte, 1982 (9): 523-532.

Leanza, H.A.; Apesteguía, S.; Novas, F.E. \& de la Fuente, M.S. (2004). Cretaceous terrestrial beds from the Neuquén Basin (Argentina) and their tetrapod assemblages. Cretaceous Research, 25 (1): 61-87. http:// dx.doi.org/10.1016/j.cretres.2003.10.005.

Lee, M.S.Y. \& Scanlon, J.D. (2002). Snake phylogeny based on osteology, soft anatomy and ecology. Biological Reviews, 77: 333-401. http://dx.doi. org/10.1017/S1464793102005924.

Linnaeus, C. (1758). Systema Naturae per Regna tria Naturae secundum Classes, Ordines, Genera, Species, cum Characteribus, Differentiis, Synonymis, Locis, volume 1. Laurenti Salvii, Stockholm, 824 pp.

Martinelli, A.G. \& Forasiepi, A.M. (2004). Late Cretaceous vertebrates from Bajo de Santa Rosa (Allen Formation), Río Negro province, Argentina, with the description of a new sauropod dinosaur (Titanosauridae). Revista del Museo Argentino de Ciencias Naturales, 6: 257-305.

Oppel, M. (1811). Die Ordnungen, Familien, und Gattungen der Reptilien als Prodom einer Naturgeschichte derselben. Joseph Lindauer Verlag, Munich, 86 pp.

Palci, A.; Caldwell, M.W. \& Albino, A.M. (2013). Emended diagnosis and phylogenetic relaonships of the Upper Cretaceous fossil snake Najash rionegrina Apesteguía and Zaher, 2006. Journal of Vertebrate Paleontology, 33: 131-140. http://dx.doi.org/10.108 $0 / 02724634.2012 .713415$.

Palci, A. \& Caldwell, M.W. (2014). The Upper Cretaceous Snake Dinilysia patagonica Smith-Woodward, 1901, and the Crista Circumfenestralis of Snakes. Journal of Morphology, 275 (10): 1187-1200. http://dx.doi. org/10.1002/jmor.20297.

Rage, J.C. (1977). La position phylétique de Dinilysia patagonica, serpent du Cretacé supérieur. Comptes Rendus de l'Académie des Sciences de Paris, 284: 1765-1768.

Rage, J.C. \& Albino, A.M. (1989). Dinilysia patagonica (Reptilia, serpents): materiel vertebral additionnel du Crétacé supérieur d'Argentine. Etude complémentaire des vertèbres, variations intraspécifiques et intracolumnaires. Neues Jahrbuch für Geologie and Paläontologie, 7: 433-447.

Rieppel, O. (1979). A cladistic classification of primitive snakes based on skull structure. Journal of Zoological 
Systematics and Evolutionary Research, 17: 140-150. http://dx.doi.org/10.1111/j.1439-0469.1979. tb00696.x.

Rieppel, O. \& Zaher, H. (2000). The intramandibular joint in squamates, and the phylogenetic relationships of the fossil snake Pachyrhachis problematicus Haas. Fieldiana Geology 43: 1-69. http://dx.doi.org/10.5962/bhl. title.3584.

Romer, A.S. (1956). Osteology of the reptiles. Chicago, University of Chicago Press, 772 pp.

Scanferla, C.A. \& Canale, J.I. (2007). The youngest record of the Cretaceous snake genus Dinilysia (Squamata, Serpentes). South American Journal of Herpetology, 2: 76-81. http://dx.doi.org/10.2994/ 1808-9798(2007)2[76:TYROTC]2.0.CO;2.

Scanferla, C.A. \& Bhullar, S.B. (2014). Posnatal development of the skull of Dinilysia patagonica (SquamataStem Serpentes). The Anatomical Record, 297: 560-573. http://dx.doi.org/10.1002/ar.22862.

Scanlon, J.D. (2006). Skull of the large non-macrostomatan snake Yurlunggur from the Australian OligoMiocene. Nature 439 (7078): 839-842. http://dx.doi. org/10.1038/nature04137.
Scanlon, J.D. \& Lee, M.S.Y. (2000). The Pleistocene serpent Wonambi and the early evolution of snakes. Nature 403 (6768): 416-420. http://dx.doi. org/10.1038/35000188.

Smith-Woodward, A. (1901). On some extinct reptiles from Patagonia, of the genera Miolania, Dinilysia and Genyodectes. Proceedings of the Zoological Society of London, 1: 169-184.

Tchernov, E.; Rieppel, O.; Zaher, H.; Polcyn, M.J. \& Jacobs, I.J. (2000). A fossil snake with limbs. Science, 287 (5460): 2010-2012. http://dx.doi.org/10.1126/ science.287.5460.2010.

Zaher, H.; Apesteguía, S. \& Scanferla, C.A. (2009). The anatomy of the upper cretaceous snake Najash rionegrina Apesteguía \& Zaher, 2006, and the evolution of limblessness in snakes. Zoological Journal of the Linnean Society, 156: 801-826. http://dx.doi. org/10.1111/j.1096-3642.2009.00511.x.

Zaher, H. \& Scanferla, C.A. (2012). The skull of the Upper Cretaceous snake Dinilysia patagonica Smith-Woodward, 1901, and its phylogenetic position revisited. Zoological Journal of the Linnean Society, 164: 194-238. http://dx.doi.org/10.1111/j.1096-3642.2011.00755.x. 